\title{
Exploring the bacterial assemblages along the human nasal passage
}

Melissa L Wos-Oxley ${ }^{1}$, Diego Chaves-Moreno ${ }^{1}$, Ruy Jáuregui ${ }^{1,2}$, Andrew P.A. Oxley ${ }^{1,3}$, Ursula Kaspar $^{4}$, Iris Plumeier ${ }^{1}$, Silke Kahl ${ }^{1}$, Claudia Rudack ${ }^{5}$, Karsten Becker ${ }^{4}$, Dietmar H Pieper ${ }^{1 *}$

${ }^{1}$ Microbial Interactions and Processes Research Group, Helmholtz Centre for Infection

Research, Inhoffenstr. 7, 38124 Braunschweig, Germany

${ }^{2}$ Present address: AgResearch, Tennent Drive, Palmerston North 4442, New Zealand

${ }^{3}$ Present address: Molecular Sciences Laboratory, SARDI Aquatic Sciences, 2 Hamra Ave,

West Beach, South Australia, Australia

${ }^{4}$ Institute of Medical Microbiology, University Hospital Münster, Münster, Germany

${ }^{5}$ Department of Otorhinolaryngology Head and Neck Surgery, University Hospital Münster, Münster, Germany

Running title: Bacterial assemblages along the nasal passage

*For correspondence: E-mail dpi@helmholtz-hzi.de; Tel. +49 53161814200 


\section{Originality-Significance Statement}

The human nasal passage is an important habitat for opportunistic pathogens and commensals. Given its unique anatomical structure and function, and that it can be prone to inflammation and a plethora of medical conditions, defining the bacterial community structure along the length of the human nasal passage is fundamental. Previous work had only marginally characterized the microbial communities of different regions with conflicting reports on the role of the microbiome in chronic inflammation. Here we compared a large cohort of individuals suffering from chronic rhinosinusitis (CRS) with individuals that were having surgery to correct for nasal deformations without inflammation. For each individual, the bacterial community structure was surveyed at four different anatomical regions along the nasal passage, allowing a deeper investigation into the role of the microbiome in health and disease. 


\begin{abstract}
The human nasal passage, from the anterior nares through the nasal vestibule to the nasal cavities, is an important habitat for opportunistic pathogens and commensals alike. This work sampled four different anatomical regions within the human nasal passage across a large cohort of individuals $(\mathrm{n}=79)$ comprising individuals suffering from chronic nasal inflammation clinically known as chronic rhinosinusitis (CRS) and individuals not suffering from inflammation (CRS-free). While individuals had their own unique bacterial fingerprint that was consistent across the anatomical regions, these bacterial fingerprints formed into distinct delineated groups comprising core bacterial members, which were consistent across all four swabbed anatomical regions irrespective of health status. The most significant observed pattern was the difference between the global bacterial profiles of swabbed and tissue biopsy samples from the same individuals, being also consistent across different anatomical regions. Importantly, no statistically significant differences could be observed concerning the global bacterial communities, any of the bacterial species or the range of diversity indices used to compare between CRS and CRS-free individuals, and between two CRS phenotypes (without nasal polyps and with nasal polyps). Thus, the role of bacteria in the pathogenesis of sinusitis remains uncertain.
\end{abstract}




\section{Introduction}

Characterizing the microbiome of the human nasal passage has held great interest in recent years. The human nasal passage encompassing the anterior nares and the vestibule comprising skin-like squamous epithelium through to the deeper nasal cavities lined with ciliated mucosa and mucus-secreting goblet cells, is considered the principle habitat of both bacterial commensals and opportunistic pathogens such as Staphylococcus aureus. S. aureus nasal carriage is asymptomatic and a crucial factor for invasive infections in both community and hospital settings (von Eiff et al., 2001). Therefore, this site has exceptional importance for strategies to detect and prevent infection and transmission by Methicillin-resistant $S$. aureus (MRSA) strains (Kock et al., 2014). While much of the focus to date has been on the anterior nares, only more recently it has been established that the nasal meatuses and the posterior vestibules are also principle habitats for S. aureus (Ramakrishnan et al., 2013; Kaspar et al., 2015).

There is now a wealth of information pertaining to the core bacterial species making up global bacterial assemblages of healthy human anterior nares (Costello et al., 2009; Grice et al., 2009; Frank et al., 2010; Wos-Oxley et al., 2010; Human Microbiome Project, 2012) and more recently of healthy human middle meatuses (Ramakrishnan et al., 2013). Seeking to shed further light onto the microbiome of the human nasal passage, recent work has endeavored to identify bacterial community differences in individuals of differing origins (Camarinha-Silva et al., 2014) and the influence of host genetics and environmental factors (Li $u$ et al., 2015), as well as age (Oh et al., 2012; Camarinha-Silva et al., 2014) and stability of the community over time (Costello et al., 2009; Grice et al., 2009; Frank et al., 2010; Camarinha-Silva et al., 2012a; Human Microbiome Project, 2012). Of particular interest has been the comparison between human anterior nares (or less commonly other nasal passage 
regions) with other skin and oral sites (Costello et al., 2009; Grice et al., 2009; Frank et al., 2010; Lemon et al., 2010; Human Microbiome Project, 2012; Bassis et al., 2014), while comparisons between specific anatomical regions of the human nasal passage have only recently been attempted (Yan et al., 2013; Biswas et al., 2015; Kaspar et al., 2015), yet within a limited number of individuals with the exception of Kaspar et al. (2015) who assessed the culturome of 34 individuals. Considering the great heterogeneity in the global bacterial communities across individuals (Camarinha-Silva et al., 2012b; Human Microbiome Project, 2012; Camarinha-Silva et al., 2014; Kaspar et al., 2015), studies comprising limited numbers of individuals do not allow formulation of statistically significant conclusions. In addition, all of these studies including those comparing persistent $S$. aureus carriers with non-carriers or non-persistent carriers (Frank et al., 2010; Yan et al., 2013) almost always involve swabbing the epithelium.

The nasal passage, which is the most superior component of the respiratory tract, has the important function of warming and humidifying inspired air, trapping and removing dust and particulate matter, and draining the paranasal sinuses and lacrimal ducts. Analyzing the global bacterial communities at the different anatomical regions along the human nasal passage is key, as the epithelium changes considerably in the short distance from the anterior and posterior vestibules comprising skin-like stratified squamous epithelium to the inferior and middle meatuses lined with ciliated pseudostratified columnar epithelium and as these regions may suffer from chronic inflammation. A few studies purport to compare the microbiome of those individuals with nasal conditions with healthy controls, particularly conditions of chronic rhinosinusitis (CRS), a heterogeneous group of sinonasal diseases associated with chronic inflammation of the nasal and the paranasal sinus mucosa which can be subdivided into 2 major phenotypes based on whether nasal polyps are present (chronic rhinosinusitis 
with nasal polyps [CRSwNP]) or absent (chronic rhinosinusitis without nasal polyps [CRSsNP]) (Van Crombruggen et al., 2010).

Previous studies of individuals suffering from CRS differ in respect to the types of samples collected, both in regards to the site of interest and the sampling technique (e.g. swabbing, brushing, lavage, mucus, aspirates or biopsy) (Power et al., 2005; Stressman $n$ et al., 2011; Feazel et al., 2012; Aurora et al., 2013; Boase et al., 2013; Bassis et al., 2014; Choi et al., 2014; Biswas et al., 2015; Kaspar et al., 2015). Thus, caution should be exercised when comparing between this broad range of studies, as the choice of sampling method may influence the recovery of different taxa (Cope and Lynch, 2015) and as the microbial composition may differ between different sites, although direct assessments to our knowledge have never been made. Nevertheless, these few studies claim that CRS patients can have a reduced diversity, altered nasal microbiota, an increase in S. aureus or Corynebacterium tuberculostearicum abundance, and greater densities of bacterial and/or fungal cells. However, the role of bacteria in the pathogenesis of CRS has yet to be established with certainty, as recent reviews argue that there is still no consensus on the most common bacteria present in the healthy or diseased state, and there is no clear causative or protective single organism (Mahdavinia et al., 2016; Ramakrishnan et al., 2016).

The current work aims to extend upon the current knowledge of the nasal microbiome, by characterizing the bacterial community structure of multiple anatomical regions of the human nasal passage, from the anterior and posterior vestibules to the inferior and middle meatuses of a greater number of individuals $(n=79)$, while also considering sampling method (swabbing vs. tissue biopsy) and existing nasal medical conditions like CRS. After obtaining a detailed bacterial fingerprint, the global bacterial profiles, the specific phylotypes-of-interest and measures for species diversity and taxonomic diversity can be compared in regards to health state, anatomical region and sampling strategy. Collectively, such an approach is both 
comprehensive and powerful for addressing the pertinent question of whether the bacterial community differs between individuals with and without CRS.

\section{Results and Discussion}

The bacterial assemblages of four anatomical regions along the human nasal passage, i.e. the anterior and posterior vestibule and the inferior and middle meatuses (see Fig. S1) were surveyed across 79 individuals (Table 1). While all four regions were swabbed in all 79 individuals, 73 of these individuals also provided tissue biopsies from both the inferior and middle meatuses. All 462 samples were analyzed by Illumina paired-end sequencing of the V1-V2 variable regions of the 16S rRNA gene. A group of 42 individuals presented with chronic rhinosinusitis (CRS), with individuals being further diagnosed as having nasal polyps (CRS with nasal polyps, CRSwNP) or not (CRS without nasal polyps, CRSsNP). For comparison, a group of 37 individuals without CRS having surgery for other reasons were included (Table 1). Other general factors recorded included age, gender, other known medical conditions, allergies and asthma, number and types of medications, smoking habits and whether they were positive for MRSA (Table 1). The only factor showing a significant difference between the CRS and CRS-free groups was age, where the median age of the CRS group was 52 and the median age of the CRS-free group was 31, as many younger individuals were having surgery to correct for nasal deformations.

Following sequencing, rarefaction analysis (Fig. S2) and standardization of library sizes, 447 phylotypes were determined and taxonomically classified. The dominant 20 phylotypes (PTs) collectively account for $>75 \%$ of the total standardized sequence reads. These PTs represent species or closely-related taxa such as Corynebacterium accolens/ 
tuberculostearicum (14.2\%), Staphylococcus epidermidis/capitis/caprae (8.3\%), Propionibacterium acnes (8.2\%), Staphylococcus aureus (7.7\%), Corynebacterium propinquum/pseudodiphtheriticum (7.2\%), Cupriavidus/Ralstonia spp. (3.9\%), Dolosigranulum pigrum (3.7\%), Enterobacteriaceae spp. (3.1\%) and Moraxella lacunata/nonliquefaciens (2.8\%) which were also among the most prevalent across the samples (Fig. S3, Dataset S1). These genera/species have all been reported to form the core bacterial community of the adult human anterior nares and of other regions of the nasal passage (Wos-Oxley et al., 2010; Human Microbiome Project, 2012; Yan et al., 2013; Camarinha-Silva et al., 2014).

Interpersonal variation

The global bacterial profiles of the four swabbed anatomical regions across the 79 individuals (a total of 316 swabbed samples) were first compared by ordination (Fig. S4). The bacterial assemblages across the nasal passage of each individual generally cluster together, while clusters of individuals are spread across the plot, with some individuals clustering closely with others, and other individuals being very different. This strong host effect is supported by the Analysis of Similarity (ANOSIM) and the Permutational Multivariate Analysis of Variation (PERMANOVA) which states that there is a strong separable and statistically significant difference between individuals overall (ANOSIM: global $\mathrm{R}=0.653$, $\mathrm{p}$-value= 0.0001, while PERMANOVA: Pseudo-F= 5.47.46, $\mathrm{P}=0.0001$ ). Such a host effect has also been observed previously across the microbiota of different sites in the nasal passage (Biswas et al., 2015; Kaspar et al., 2015), where more of the variation in bacterial composition could be explained by interpersonal differences, rather than sampling location or even health status. 
However, while host genetics is known to play a significant role in shaping bacterial communities in other host-associated habitats like the gut (Khachatryan et al., 2008; Li and Hotamisligil, 2010; Wos-Oxley et al., 2012), a recent study comparing twins suggested that the nasal microbiota is not fixed by host genetics but is an environmentally derived host phenotype (Liu et al., 2015).

Delineation of groups sharing similar nasal assemblages and comparisons between anatomical regions

Although there was a strong host effect, delineated groups of individuals with similar nasal community structures exist. At each swabbed anatomical region, the 79 individuals could be statistically classified into 13-17 delineated groups (Fig. 1), where individuals share the same bacterial PTs at similar relative abundances. Most individuals collapsed into just a few predominant groups, defined as groups comprising $>7$ individuals. Interestingly, these predominant delineated groups at each of the four nasal passage regions were consistently composed of the same taxa (Fig. 2). The predominant group across all regions comprised individuals with high levels of C. accolens/tuberculostearicum (PT 1), alongside moderate levels of both P. acnes (PT 2) and S. epidermidis/capitis/caprae (PT 4). The second predominant group comprised individuals with high levels of C. propinquum/pseudodiphtheriticum (PT 3) and D. pigrum (PT 6) with lower levels of C. accolens/tuberculostearicum (PT 1) and the third predominant group comprised individuals with high levels of S. aureus (PT 5) and only moderate-low levels of C. accolens/tuberculostearicum (PT 1), $P$. acnes (PT 2) and S. epidermidis/capitis/caprae (PT 4). Other smaller groups of individuals $(<7)$ were dominated by high relative abundances of other important bacterial taxa of the 
nasal passage, like Moraxella spp., F. magna, and Prevotella spp. along with various other equally abundant taxa such as $S$. epidermidis or $H$. influenzae. This implies that there are core bacterial community profiles within the human nasal passage, as has been previously reported for the human anterior nares (Camarinha-Silva et al., 2012a; Liu et al., 2015) and more recently for the middle meatuses (Ramakrishnan et al., 2013). Camarinha-Silva and colleagues reported that 100 anterior nares microbial communities from healthy adults could be statistically clustered into 12 groups, where two thirds of individuals shared more than $40 \%$ similarity in respect to their bacterial community structure, as being dominated by organisms like Corynebacterium spp., P. acnes, uncultured Actinomycetales and $S$. epidermidis as well as Peptoniphilus spp. and F. magna, while the remaining third of individuals showed communities which clustered together in smaller groups being dominated by either D. pigrum, Moraxella spp. or S. aureus (Camarinha-Silva et al., 2012b). Later, Liu and colleagues similarly identified seven major groups (labeled as community state types, CST) among 178 anterior nares samples (Liu et al., 2015), where likewise, each group comprised a high prevalence and proportional abundance of either $S$. aureus, members of the Enterobacteriaceae family, S. epidermidis, Propionibacterium spp., Corynebacterium spp., Moraxella spp. or Dolosigranulum spp. Similar concepts of demarcating samples into "types" have been applied to other human habitats like the gut (Arumugam et al., 2011), where it was reported that the intestinal community structure of humans can be delineated into three "enterotypes" based on the abundance of either Bacteroides, Prevotella and/or Ruminococcus species. It was then suggested that the limited number of well-balanced host-microbial symbiotic states might respond differently to diet and drug intake (Arumugam et al., 2011). This begs the question of whether an individual belonging to a particular group, CST or enterotype might respond differently to antibiotic treatment or another intervention for a nasal medical condition, or if the likely success of an individual for a particular treatment is 
governed by its "type" classification. Importantly, these core bacterial community profiles are consistent across the spatial regions of the nasal passages.

Formal comparisons between the global bacterial assemblages across the four different regions of the human nasal passage reveal that the bacterial structures were indeed not separable or significantly different (ANOSIM: global $\mathrm{R}=0.005, \mathrm{p}$-value $=0.108$;

PERMANOVA: Pseudo-F=1.3168, p-value= 0.0956). Furthermore, comparing measures of species diversity, species richness, species evenness and taxonomic distinctness, or speciesspecific abundances between swab samples of these anatomical regions did not yield any further patterns or any statistical differences (Fig. S3, Fig. S5, p > 0.05 in all comparisons). This unchanging bacterial community profile may reflect the "open" nature of the human nasal passage, and may signify the influence of mucus, which covers the respiratory tract and drains through the nasal passage into the nasopharynx. In this aspect, the nasal passage is clearly different to the oral cavity where highly distinct habitats have been described (Aas et al., 2005).

Comparing the bacterial assemblages between tissue biopsies and swabs

Comparison of the global bacterial communities obtained by swabbing and tissue biopsy at the same anatomical regions, revealed that the bacterial community structures were significantly different and statistically separable at both of the two independent regions of the meatuses (middle meatus: PERMANOVA Pseudo-F= 5.548, $\mathrm{p}$-value $=0.0001$; ANOSIM global $\mathrm{R}=0.084, \mathrm{p}$-value $=0.0001, \mathrm{n}=73$; inferior meatus: PERMANOVA Pseudo- $\mathrm{F}=12.08$, $\mathrm{p}$-value $=0.0001 ;$ ANOSIM global $\mathrm{R}=0.195, \mathrm{p}$-value $=0.0001, \mathrm{n}=73$, see Fig. 3 ). Of the 447 phylotypes, only between 14-20 contributed towards $70 \%$ of the observed difference between 
swabbing and tissue biopsies of the middle meatus and the inferior meatus between CRS and CRS-free groups (listed in Table S1, using the similarity percentage algorithm). Of these phylotypes, only P. acnes (PT 2), C. accolens/tuberculostearicum (PT 1) and S. epidermidis/capitis/caprae (PT 4) were more abundant in the swabbed samples whereas Cupriavidus/Ralstonia (PT 7), R. pickettii (PT 11) and Enterobacteriaceae (PT 13) were generally more abundant in the tissue samples (Table S1).

Interestingly, for both meatus regions, the tissue biopsies recovered greater species richness and diversity with greater evenness of phylotype abundance and less dominance of a few phylotypes, as can be seen using k-dominance curves (Fig. S6). The average species richness was $94.3 \pm 40.6$ and $82.7 \pm 39.8$ PTs per tissue sample for the middle meatus and inferior meatus, respectively, while only $69.8 \pm 37.1$ and $58.4 \pm 35.1$ PTs were observed when analyzing the respective swab samples (Fig. 4). The difference was statistically highly significant (Welsh's t-test for middle meatus, $\mathrm{t}=3.892, \mathrm{p}=0.0002$; for inferior meatus, $\mathrm{t}=$ $3.976, p=0.0001)$. This difference is also evident by a range of other diversity indices that clearly show that the tissue samples returned higher values for species diversity (using Shannon and Simpson indices), species richness (using Margelef's indices), species evenness (using Pielou's index) and taxonomic distinctness (delta metrics and lambda + ) (see Fig. S5). Formal hypothesis testing of these diversity indices (using both the Kruskal-Wallis test with Dunn's multiple comparison posthoc test and the Welsh's t-test assuming unequal variances) confirmed that these measures of species diversity were significantly different between the tissue and swabbed groups of samples (all $p<0.05$ ). To further explore for patterns in taxonomic diversity, average taxonomic distinctness (delta + ) and variation in taxonomic distinctness (lambda + ) were plotted against the number of PTs for each sample (Fig. 4). A greater proportion of samples derived from swabbing appear to be below the 'expected range' (depicted as the funnel lines in Fig. 4), indicating low taxonomic breadth and greater 
taxonomic unevenness per sample than the tissue biopsies. In contrast, most of the tissue biopsy samples returned delta + and lambda + values that fell within or closer to the expected range. This observation was confirmed, as there was a significant difference in measures of taxonomic diversity (delta + and lambda + ) between tissue and swab samples at both the middle meatus and inferior meatus regions (Welsh's t-test for middle meatus for delta,$+ \mathrm{t}=$ 2.284, $\mathrm{p}$-value $=0.0238$ and for lambda $+, \mathrm{t}=3.678, \mathrm{p}$-value $=0.0004$; for inferior meatus for delta $+, \mathrm{t}=3.508, \mathrm{p}$-value $=0.0006$ and for lambda $+, \mathrm{t}=4.186, \mathrm{p}$-value $=<0.0001)$. In contrast to the observed difference in bacterial profiles obtained from tissue biopsy and swabbing, the only other study to have compared tissue biopsies with swabbing technique, albeit of the ethmoid sinuses and across only 6 individuals, indicated that the sampling method did not yield significant differences in the phylogenetic and non-phylogenetic alpha and beta diversity metrics (Bassiouni et al., 2015). Given the large inter-individual variation in the bacterial assemblages, obviously a greater number of sampled individuals is needed to observe such a difference between sampling methods, as is seen here using 73 individuals, being consistent across both anatomical regions analyzed. This raises the point that the sampling strategy has an impact on the discernible bacterial fingerprint, where swabbing would more likely target those bacterial loosely associated to the epithelial surfaces, while tissue biopsies target also strongly adhering cells. There are of course advantages to swabbing, as it is non-destructive and that most studies of the anterior nares and other related habitats have used swabbing as a method for sample collection, while tissue biopsy has the advantage of being able to capture the entire site of interest but is invasive. This again emphasizes the dilemma of comparing different studies that may have sampled differently, as sampling technique may influence the recovery of different taxa (Cope and Lynch, 2015). 
Comparing the bacterial assemblages between groups of CRS and CRS-free individuals

Comparisons of the global bacterial community structures of those individuals clinically diagnosed with CRS (including both CRSwNP and CRSsNP), with those of individuals without inflammation indicated no statistical significant differences between the groups at each of the 4 sites (PERMANOVA: $p$-value $=>0.05, n=37$ for CRS-free group and $n=42$ for CRS group), as evident in Fig. 5A. Comparison of the profiles of individuals with CRSsNP and those with CRSwNP at each of the 4 sites using both sampling strategies indicated no significant differences in community structures between both clinical presentations (of the 6 comparisons, PERMANOVA Pseudo-F ranged between 0.85-1.45, p-values ranged from 0.111-0.614) (Fig. 5B). Differential abundance analysis was performed on each phylotype-ofinterest. No phylotype was deemed to be statistically significantly different in relative abundance between the groups of CRS-free and CRS individuals, or between groups of CRSsNP and CRSwNP individuals.

To rule out that other factors such as age, use of steroids and/or suffering from allergic rhinitis and/or asthma may impact these findings, the global bacterial profiles of subsets of individuals were further compared using the PERMANOVA test. As age was deemed the only factor to significantly differ between the CRS-free and CRS groups (see Table 1), the communities of younger individuals (18-40 years of age) from the CRS group $(n=16)$ were compared to communities of the same age-class of the CRS-free group $(n=23)$, however no significant differences between the global bacterial profiles were observed. Similarly, neither a comparison of communities of 19 individuals within the CRS group that were reportedly to not be using any medication with those 24 individuals from the CRS-free group, nor a comparison of the 28 individuals within the CRS group that were reportedly free from allergic rhinitis and asthma with those 25 individuals from the CRS-free group gave any indication for 
significant differences between global bacterial profiles at the sampling sites. This was repeated for the CRSsNP and CRSwNP groups, and no further significant differences were found. This indicates that age, use of medications or suffering from allergic rhinitis or asthma have no influence on the observation that there are no differences between the global bacterial profiles of CRS and CRS-free individuals. Furthermore, the derived groupings of individuals seen in Fig. 1 could not be associated to a disorder or any other factors tested in this work.

Comparisons of phylotype diversity, richness, evenness, dominance and taxonomic distinctness between samples from CRS-free and CRS individuals, or between CRSsNP and CRSwNP individuals did not yield any further patterns or any statistical differences (Fig. S5, $\mathrm{p}>0.05$ for all comparisons). In particular, individuals within both the CRS-free group of individuals and the group of individuals suffering from CRS have a wide range of values for taxonomic distinctness that fall within and outside of the expected range (Fig. 6). This thus suggests that chronic rhinosinusitis, does not result in a reduced phylotype richness or taxonomic diversity, as may have been expected (Wilson and Hamilos, 2014).

A few studies have reported a reduction in bacterial community diversity in CRS patients, such as in those obtained from brushings of the maxillary sinuses (comparing $n=10 \mathrm{CRS}$ individuals with $\mathrm{n}=10$ controls) (Abre $u$ et al., 2012), from swabbed samples of 3 different regions within the nasal passage (comparing $n=9$ CRS individuals with $n=6$ controls) (Biswas et al., 2015), from lavage of the nasal cavity (comparing $n=8$ CRS individuals with $n=3$ controls) (Choi et al., 2014), however all using restricted sample sizes. Liu and colleagues reported that there was a significant decrease in microbiota diversity and evenness from swabs of the maxillary sinuses $(n=6)$, their statistics contrast this conclusion (with non-significant $p$ values), and they also report that there was no single common microbiota profile among participants with similar clinical outcomes (Li $u$ et al., 2013). Other studies report that patients with CRS have altered nasal microbiota (Feazel et al., 2012; Choi et al., 2014) and/or an 
increase in S. aureus abundance (Feazel et al., 2012; Boase et al., 2013; Choi et al., 2014) or a decrease in multiple phylogenetically distinct lactic acid bacteria with an increase in the relative abundance of C. tuberculostearicum (Abreu et al., 2012). Although overshadowing this result is the fact that 6 of their $10 \mathrm{CRS}$ patients were receiving preoperative antibiotic treatment. In contrast, other studies report that patients with CRS do not necessarily have altered phylogenetic composition. For example, a study using lavage of the middle meatus (comparing $\mathrm{n}=30$ CRS individuals with $\mathrm{n}=12$ controls) (Aurora et al., 2013) reports that bacteria and fungi do not trigger CRS, as the microbiomes of patients with CRS were qualitatively similar to the controls. The authors indicated that in some cases CRS results from an immune hyper-responsiveness to commensal organisms (Aurora et al., 2013), whereby CRS patients may have greater densities of bacterial and/or fungal cells (Aurora et al., 2013; Boase et al., 2013). Another study comparing the bacterial cultures from the swabs of the middle meatus from CRSsNP and CRSwNP patients with a control group, reported that while there was a higher rate of cultivable pathogenic bacteria in patients with more severe forms of CRS, there were no differences in bacterial species between patients presenting with CRSwNP, CRSsNP or control groups (Uhliarova et al., 2014). However, in contrary again, Biswas and colleagues found that bacterial load was not associated with CRS (Biswas et al., 2015). While bacterial density was not measured in this present work, our results might suggest that if bacterial density increases under a particular condition like CRS, it is not associated with just an overgrowth of a single species like $S$. aureus, as there was no trend of dysbiosis in community structure across the CRS cohort analyzed here.

Thus, it is clear that there are conflicting reports of the role of the microbiome in CRS, however, these differences are likely due to intrapersonal variation of the sinus/nasal microbiota shaped by environmental exposures and geography, as well as the variation in sampling techniques and approaches to assay and analyze the microbiome (Cope and Lynch, 
2015). Moreover, the conflicting results are in addition due to the small sample sizes that most studies have published on and their likely lack of statistical power to make true inference pertaining to CRS and control groups. Accordingly, recent thorough reviews of the literature (Cope and Lynch, 2015; Mahdavinia et al., 2016; Ramakrishnan et al., 2016) conclude that the existing data do not provide a consensus on the role of the bacterial community in relation to CRS. This new work here demonstrates with a greater number of individuals that the role of bacteria in the pathogenesis of CRS has still yet to be established with certainty.

\section{Conclusions}

While individuals have their own unique bacterial fingerprint, there are still delineated groups of individuals that share a core bacterial community as evident here for regions of the nasal passage. Importantly these groups comprising core bacterial members are consistent along the epithelium of the nasal passages, from the anterior vestibule to the middle meatus. The existence of a limited number of host-microbial symbiotic states begs the question of whether the response of an individual to a particular treatment like antibiotics or to biological or environmental stimuli is correlated to its "type" classification. The bacterial composition did not significantly change along the nasal passage. There were no differences between global bacterial assemblages, measures of diversity or any of the specific nasal-associated phylotypes between the four different anatomical regions, in lieu of a change in epithelium structure to which the bacteria are associated. However, there were highly significant differences between the bacterial assemblages and diversity measures observed for different sample types, i.e. between tissue biopsies and swabs. In contrast, no significant differences could be observed between the global bacterial communities of groups of CRS-free individuals with those that suffer from CRS. 


\section{Experimental procedures}

\section{Sampling strategy}

This study was designed to compare the bacterial community structure along the human nasal passage obtained using different sampling strategies (swabbing versus tissue biopsy) and between groups of individuals suffering from CRS. This cross-sectional study was conducted with full written consent of patients (file number 2010-468-f-S) admitted to the Department of Otorhinolaryngology Head and Neck Surgery (ENT) of the University Hospital Münster. Inclusion criteria included adult ( $>18$ years of age) individuals having been clinically diagnosed with CRS according to the criteria defined by the European position paper on rhinosinusitis and nasal polyps guidelines (Fokkens WJ, 2012) or individuals undergoing surgery to correct for nasal deformations. Exclusion criteria included (i) taking antibiotics or oral immunosuppressants at the time of sampling and/or (ii) having been diagnosed and having surgery for a melanoma or papilloma. A group of 42 individuals presented with clinically diagnosed CRS, defined by the presence of at least two of the following symptoms: nasal obstruction, nasal secretion and/or post-nasal drip, headaches and/or facial pains. All patients had a CT- scan to verify or to exclude CRS. Endoscopic analysis subdivided this group into 2 major phenotypes based on whether nasal polyps are present (chronic rhinosinusitis with nasal polyps, CRSwNP, $n=15$ ) or absent (chronic rhinosinusitis without nasal polyps, CRSsNP, $n=27)$. Sinus healthy patients serving as controls in this study $(n=37)$ were classified as healthy by CT-scan excluding radiographical signs of rhinosinusitis. The pathology of the nose in these cases comprises septal deviations, hypertrophy of the inferior 
tubinates and nasal bone fracture or septal deviations.

In total, 79 individuals provided swab samples at four different regions along the nasal passage (anterior and posterior vestibules and inferior and middle meatuses, see Fig. S1) using SK-2S DNA swabs (Isohelix, Harrietsham, United Kingdom) and of these 73 also provided tissue biopsies of the inferior and middle meatuses. For swabbing the vestibules, an experienced surgeon uses a speculum and a retractor to recognize the stratified squamous, keratinized epithelium containing vibrissae and then swabs separately the anterior and posterior parts of the vestibules. For swabs and tissue biopsies of the inferior and middle meatuses, samples were obtained during routine surgery at the ENT using a speculum to avoid bacterial contamination by adjacent nasal sites during tissue/swab removal as previously described (Sachse et al., 2010). In brief, the vestibular skin and the mucocutaneous junction area were disinfected using swabs of Octenisept solution and then a sterile self-fixing nasal speculum was used to create enough space for a contact-free removal of nasal tissue/swab using sterile instruments (Sachse et al., 2010). All samples were immediately stored at $-20{ }^{\circ} \mathrm{C}$ until further analysis.

Several key factors were recorded for each individual (such as age, gender, diagnosed medical condition of the nose, known allergies and asthma, types and number of medications and smoking habits and whether they were positive for MRSA).

DNA extraction, amplicon library preparation and sequencing

DNA was extracted from the nasal swabs and the tissue biopsies using the FastDNA Spin Kit for Soil (MP Biomedicals, Solon, OH, USA) following the manufacturer's instructions. The bacterial $16 \mathrm{~S}$ rDNA amplicon libraries were prepared for Illumina deep-sequencing on the 
MiSeq platform (Illumina, San Diego, CA) using 250 nt paired-end sequencing chemistry. Samples were randomly pooled and run across 6 MiSeq runs. The V1-2 region of the $16 \mathrm{~S}$ rRNA gene was amplified as previously described (Camarinha-Silva et al., 2014). However, in a first 20 cycle PCR reaction the $16 \mathrm{~S}$ rDNA target was enriched using the previously described 27F and 338R primers (Chaves-Moreno et al., 2015). One $\mu 1$ of this reaction mixture served as template in a second 15 cycle PCR where the forward primer contains a 6 nucleotide (nt) barcode and a 2 nt CA linker and where both primers comprised sequences complementary to the Illumina specific adaptors to the 5 '-ends. One $\mu 1$ of this reaction mixture served as template in a third 10 cycle PCR using PCR primers designed to integrate the sequence of the specific Illumina multiplexing sequencing primers and index primers (Camarinha-Silva et al., 2014).

\section{Bioinformatic and statistical analysis}

Around 8 million sequence reads were derived from 462 samples. These were subsequently quality-checked, filtered, trimmed, paired and collapsed as previously described (CamarinhaSilva et al., 2014). For this study, $80 \mathrm{nt}$ of the forward and $80 \mathrm{nt}$ of the reverse sequence reads were combined to form a $160 \mathrm{nt}$ sequence, which was used for clustering allowing for 2 mismatches. The appropriate 3 million good-quality paired-end sequence reads were collapsed into 450 bacterial phylotypes (PTs). The average sequence depth per sample was 7,192 reads (+/- 7,020 S.D; min of 877 reads; max of 36,355 reads). Considering the variation in library sizes, libraries were first standardized by random resampling to 2000 reads taking the consensus from 9999 iterations (Szafransk $a$ et al., 2014) following inspection of rarefaction curves (Fig. S2). While $85 \%$ of the samples comprised $>2000$ sequence reads only 
5 samples had just under 1000 reads. In case of low sequence read numbers, the rarefaction curves were manually inspected and only kept in the dataset if deemed to have reached a plateau (see Fig. S2). All 447 PTs remaining after resampling (see Datasets S1 and S2) were assigned a taxonomic affiliation based on naïve Bayesian classification (RDP classifier) (Wang et al., 2007). PTs were then manually analyzed against the RDP database using the Seqmatch function as well as against the nucleotide collection (nr/nt) database at NCBI to define the discriminatory power of each sequence read. A species name was assigned to a phylotype when only 16S rRNA gene fragments of previously described isolates of that species showed $\leq 2$ mismatches with the respective representative sequence read.

In order to explore for patterns across the global bacterial communities, the data matrix comprising the percent standardized abundances of 447 PTs was used to construct a samplesimilarity matrix using the Bray-Curtis algorithm (Bray and Curtis, 1957), where samples were then ordinated using non-metric multidimensional scaling (nMDS) with 50 random restarts (Clarke et al., 2001). Significant differences between a priori predefined groups of samples were evaluated using Analysis of Similarity (ANOSIM) (9999 permutations) with the accompanying R statistic measuring the degree of separation between groups (Clarke et al., 2001) and/or permutational multivariate analysis of variance (PERMANOVA), allowing for; type III (partial) sums of squares, fixed effects sum to zero for mixed terms, and exact pvalues generated using unrestricted permutation of raw data (Anderson, 2001). Groups of samples were considered significantly different if the $\mathrm{p}$-value falls $<0.05$. When a significant difference was found between a priori groups, the Similarity Percentage (SIMPER) routine was applied to seek for those PTs that contribute mostly to this observed difference (Clarke et al., 2001). Delineated groups of individuals with similar nasal community structures were determined by using the Similarity Profile permutation test (SIMPROF) associated with group-average agglomerative hierarchical clustering, using significance level of 1\%; 1,000 
permutations for mean profile; 999 simulation permutations, where statistically significant evidence of genuine clusters in the absence of a priori groups could be sought (Clarke et al., 2008). Like with the nMDS analysis, the subsequent ANOSIM, PERMANOVA, SIMPER and SIMPROF routines also used the Bray-Curtis similarity algorithm.

Species diversity, richness and evenness were calculated using algorithms for total phylotypes (S), Margelef's richness (d), Shannon diversity (H'), Simpson (1- $\lambda$ ) and Pielou's evenness $\left(\mathrm{J}^{\prime}\right)$, while taxonomic diversity was calculated using algorithms for taxonomic diversity (delta), taxonomic distinctness (delta*), average taxonomic distinctness (delta + ), total taxonomic distinctness (sDelta + ) and variation in taxonomic distinctness (lambda + ) (Clarke et al., 2001). Delta + represents the average taxonomic distance between all pairs of species within each sample, and thus is a summary of average taxonomic breadth of each sample, while lambda+ reports how consistent each level of organization within the Linnaean classification is represented (Warwick and Clarke, 1995; Pienkowski et al., 1998). The expected delta + values were calculated by sampling different numbers of PTs (from 5 to 250 in increments of 10) from the master list of 447 PTs, repeating 999 times. Values for delta+ and lambda + are plotted against the number of PTs within each sample and compared to those 'expected values' derived from a species master list ascertained across all samples, where the funnel indicates the limits within $95 \%$ of the simulated taxonomic distinctness (TD) values and the middle line represents the mean expected TD, thus providing a statistical framework to test whether these measures depart from expectation (Warwick and Clarke, 1995; Pienkowski et al., 1998).

The abundances of phylotypes-of-interest and univariate diversity indices were compared between a priori groups of samples using either a t-test or an ANOVA style test. First, each variable of interest was subjected to a normality test using both the D'Agostino \& Pearson omnibus and the Shapiro-Wilk algorithms (Prism 5, Graphpad Software Inc). Since most of the 
PT abundances across most of the groups returned estimates indicating non-normal distributions, non-parametric versions of the t-test and ANOVA were performed. The Kruskal-Wallis test with Dunn's multiple comparison posthoc test was used when multiple groups were being compared whereas the Mann-Whitney test was used when only 2 groups were compared. T-tests allowing for unequal variances (Welsh's t-test) were also chosen in some cases. When allowing for multiple testing as multiple PTs were assessed, both the False Discovery Rate (FDR) approach allowing a desired FDR (Q) of either $1 \%$ or $5 \%$, and the Holm-Sidak correction method $($ alpha $=0.05)$ were independently applied. Groups of samples were considered significantly different if the p-value falls $<0.05$.

The factors ascribed for each individual in the study (such as age, gender, diagnosed medical condition of the nose, known allergies and asthma, types and number of medications and smoking habits) were used to test for differences between CRS and CRS-free groups using either the Mann-Whitney test (two-tailed, unpaired), the unpaired t-test assuming equally variance (two-tailed) or not assuming equal variance (with Welch's correction), the Fisher's exact test (two-tailed, confidence intervals at 95\%) or the Chi-square test (confidence intervals at 95\%) depending upon the factor being assessed (see Table 1 for specific details).

The multivariate analyses were performed and diversity indices were calculated using PRIMER (v.6.1.6, PRIMER-E, Plymouth Marine Laboratory, UK, (Clarke et al., 2001), while the univariate analyses were performed in Prism 5 (Graphpad Software, Inc.).

\section{Acknowledgements}

This work was supported by a grant from the German Ministry for Education and Research (BMBF) to DP (0315832B) and KB and CR (0315832A) within the project 'The Microbiota of the Human Nose Habitats - Metagenomic Analyses of their Composition and Dynamics' of 
the 'Medizinische Infektionsgenomik' call. 


\section{References}

Aas, J.A., Paster, B.J., Stokes, L.N., Olsen, I., and Dewhirst, F.E. (2005) Defining the normal bacterial flora of the oral cavity. J Clin Microbiol 43: 5721-5732.

Abreu, N.A., Nagalingam, N.A., Song, Y., Roediger, F.C., Pletcher, S.D., Goldberg, A.N., and Lynch, S.V. (2012) Sinus microbiome diversity depletion and Corynebacterium tuberculostearicum enrichment mediates rhinosinusitis. Sci Trans1 Med 4: 151 ra124. Anderson, M.J. (2001) A new method for non-parametric multivariate analysis of variance. Austral Ecol 26: 32-46.

Arumugam, M., Raes, J., Pelletier, E., Le Paslier, D., Yamada, T., Mende, D.R. et al. (2011) Enterotypes of the human gut microbiome. Nature 473: 174-180.

Aurora, R., Chatterjee, D., Hentzleman, J., Prasad, G., Sindwani, R., and Sanford, T. (2013) Contrasting the microbiomes from healthy volunteers and patients with chronic rhinosinusitis. JAMA Otolaryngol Head Neck Surg 139: 1328-1338.

Bassiouni, A., Cleland, E.J., Psaltis, A.J., Vreugde, S., and Wormald, P.-J. (2015) Sinonasal microbiome sampling: a comparison of techniques. PloS One 10: e0123216.

Bassis, C.M., Tang, A.L., Young, V.B., and Pynnonen, M.A. (2014) The nasal cavity microbiota of healthy adults. Microbiome 2: 27.

Biswas, K., Hoggard, M., Jain, R., Taylor, M.W., and Douglas, R.G. (2015) The nasal microbiota in health and disease: variation within and between subjects. Front Microbiol 9: 134.

Boase, S., Foreman, A., Cleland, E., Tan, L., Melton-Kreft, R., Pant, H. et al. (2013) The microbiome of chronic rhinosinusitis: culture, molecular diagnostics and biofilm detection. BMC Infect Dis 13: 210. 
Bray, J.R., and Curtis, J.T. (1957) An ordination of the upland forest communities of southern Wisconsin. Ecol Monogr 27: 325-349.

Camarinha-Silva, A., Jáuregui, R., Pieper, D.H., and Wos-Oxley, M.L. (2012a) The temporal dynamics of bacterial communities across human anterior nares. Environ Microbiol Rep 4: 126-132.

Camarinha-Silva, A., Wos-Oxley, M.L., Jauregui, R., Becker, K., and Pieper, D.H. (2012b) Validating T-RFLP as a sensitive and high-throughput approach to assess bacterial diversity patterns in human anterior nares. FEMS Microbiol Ecol 79: 98-108.

Camarinha-Silva, A., Jáuregui, R., Chaves-Moreno, D., Oxley, A.P.A., Schaumburg, F., Becker, K. et al. (2014) Comparing the anterior nare bacterial community of two discrete human populations using Illumina amplicon sequencing. Environ Microbiol 16: 2939-2952.

Chaves-Moreno, D., Plumeier, I., Kahl, S., Krismer, B., Peschel, A., Oxley, A.P. et al. (2015) The microbial community structure of the cotton rat nose. Environ Microbiol Rep 7: 929-935. Choi, E.B., Hong, S.W., Kim, D.K., Jeon, S.G., Kim, K.R., Cho, S.H. et al. (2014) Decreased diversity of nasal microbiota and their secreted extracellular vesicles in patients with chronic rhinosinusitis based on a metagenomic analysis. Allergy 69: 517-526.

Clarke, K.R., Warwick, R.M.R.M., and Laboratory, P.M. (2001) Change in marine communities : an approach to statistical analysis and interpretation: Plymouth, U.K. : PRIMER-E Ltd.

Clarke, K.R., Somerfield, P.J., and Gorley, R.N. (2008) Testing of null hypotheses in exploratory community analyses: similarity profiles and biota-environment linkage. J Exp Mar Biol Ecol 366: 56-69.

Cope, E.K., and Lynch, S.V. (2015) Novel microbiome-based therapeutics for chronic rhinosinusitis. Curr Allergy Asthma Rep 15: 504. 
Costello, E.K., Lauber, C.L., Hamady, M., Fierer, N., Gordon, J.I., and Knight, R. (2009)

Bacterial community variation in human body habitats across space and time. Science 326: 1694-1697.

Feazel, L.M., Robertson, C.E., Ramakrishnan, V.R., and Frank, D.N. (2012) Microbiome complexity and Staphylococcus aureus in chronic rhinosinusitis. Laryngoscope 122: 467-472. Fokkens WJ, L.V., Mullol J, Bachert C, Alobid I, Baroody F, Cohen N et al (2012) European position paper on rhinosinusitis and nasal polyps 2012. A summary for otorhinolaryngologists. Rhinol 50: 1-12.

Frank, D.N., Feazel, L.M., Bessesen, M.T., Price, C.S., Janoff, E.N., and Pace, N.R. (2010) The human nasal microbiota and Staphylococcus aureus carriage. PLoS ONE 5: e10598. Grice, E.A., Kong, H.H., Conlan, S., Deming, C.B., Davis, J., Young, A.C. et al. (2009) Topographical and temporal diversity of the human skin microbiome. Science 324: 11901192.

Human Microbiome Project, C. (2012) Structure, function and diversity of the healthy human microbiome. Nature 486: 207-214.

Kaspar, U., Kriegeskorte, A., Schubert, T., Peters, G., Rudack, C., Pieper, D.H. et al. (2015) The culturome of the human nose habitats reveals individual bacterial fingerprint patterns. Environ Microbiol 10.1111/1462-2920.12891.

Khachatryan, Z.A., Ktsoyan, Z.A., Manukyan, G.P., Kelly, D., Ghazaryan, K.A., and Aminov, R.I. (2008) Predominant role of host genetics in controlling the composition of gut microbiota. PLoS ONE 3: e3064.

Kock, R., Becker, K., Cookson, B., van Gemert-Pijnen, J.E., Harbarth, S., Kluytmans, J. et al. (2014) Systematic literature analysis and review of targeted preventive measures to limit healthcare-associated infections by meticillin-resistant Staphylococcus aureus. Euro Surveill 19. 
Lemon, K.P., Klepac-Ceraj, V., Schiffer, H.K., Brodie, E.L., Lynch, S.V., and Kolter, R.

(2010) Comparative analyses of the bacterial microbiota of the human nostril and oropharynx. mBio 1: e00129-00110.

Li, P., and Hotamisligil, G.S. (2010) Metabolism: Host and microbes in a pickle. Nature 464: $1287-1288$.

Liu, C.M., Soldanova, K., Nordstrom, L., Dwan, M.G., Moss, O.L., Contente-Cuomo, T.L. et al. (2013) Medical therapy reduces microbiota diversity and evenness in surgically recalcitrant chronic rhinosinusitis. Int Forum Allergy Rhinol 3: 775-781.

Liu, C.M., Price, L.B., Hungate, B.A., Abraham, A.G., Larsen, L.A., Christensen, K. et al. (2015) Staphylococcus aureus and the ecology of the nasal microbiome. Sci Adv 1: e1400216. Mahdavinia, M., Keshavarzian, A., Tobin, M.C., Landay, A.L., and Schleimer, R.P. (2016) A comprehensive review of the nasal microbiome in chronic rhinosinusitis (CRS). Clin Exp Allergy 46: 21-41.

Oh, J., Conlan, S., Polley, E.C., Segre, J.A., and Kong, H.H. (2012) Shifts in human skin and nares microbiota of healthy children and adults. Genome Med 4: 77.

Pienkowski, M.W., Watkinson, A.R., Kerby, G., Clarke, K.R., and Warwick, R.M. (1998) A taxonomic distinctness index and its statistical properties. J Appl Ecol 35: 523-531. Power, D.A., Burton, J.P., Chilcott, C.N., Tagg, J.R., and Dawes, P.J. (2005) Non-culturebased analysis of bacterial populations from patients with chronic rhinosinusitis. J Clin Microbiol 43: 5822-5824.

Ramakrishnan, V.R., Hauser, L.J., and Frank, D.N. (2016) The sinonasal bacterial microbiome in health and disease. Curr Opin Otolaryngol Head Neck Surg 24: 20-25.

Ramakrishnan, V.R., Feazel, L.M., Gitomer, S.A., Ir, D., Robertson, C.E., and Frank, D.N. (2013) The microbiome of the middle meatus in healthy adults. PLoS ONE 8: e85507. 
Sachse, F., Becker, K., von Eiff, C., Metze, D., and Rudack, C. (2010) Staphylococcus aureus invades the epithelium in nasal polyposis and induces IL-6 in nasal epithelial cells in vitro. Allergy 65: 1430-1437.

Stressmann, F.A., Rogers, G.B., Chan, S.W., Howarth, P.H., Harries, P.G., Bruce, K.D., and Salib, R.J. (2011) Characterization of bacterial community diversity in chronic rhinosinusitis infections using novel culture-independent techniques. Am J Rhinol Allergy 25: e133-140. Szafranska, A.K., Oxley, A.P., Chaves-Moreno, D., Horst, S.A., Rosslenbroich, S., Peters, G. et al. (2014) High-resolution transcriptomic analysis of the adaptive response of Staphylococcus aureus during acute and chronic phases of osteomyelitis. MBio 5. Uhliarova, B., Karnisova, R., Svec, M., and Calkovska, A. (2014) Correlation between culture-identified bacteria in the middle nasal meatus and CT score in patients with chronic rhinosinusitis. J Med Microbiol 63: 28-33.

Van Crombruggen, K., Van Bruaene, N., Holtappels, G., and Bachert, C. (2010) Chronic sinusitis and rhinitis: clinical terminology "Chronic Rhinosinusitis" further supported. Rhinology 48: 54-58.

von Eiff, C., Becker, K., Machka, K., Stammer, H., and G., P. (2001) Nasal carriage as a source of Staphylococcus aureus bacteremia. N Engl J Med 344: 11-16.

Wang, Q., Garrity, G.M., Tiedje, J.M., and Cole, J.R. (2007) Naive Bayesian classifier for rapid assignment of rRNA sequences into the new bacterial taxonomy. Appl Environ Microbiol 73: 5261-5267.

Warwick, R., and Clarke, K. (1995) New 'biodiversity' measures reveal a decrease in taxonomic distinctness with increasing stress. Mar Ecol Prog Ser 129: 301-305. Wilson, M.T., and Hamilos, D.L. (2014) The nasal and sinus microbiome in health and disease. Curr Allergy Asthma Rep 14: 485. 
Wos-Oxley, M., Bleich, A., Oxley, A.P., Kahl, S., Janus, L.M., Smoczek, A. et al. (2012) Comparative evaluation of establishing a human gut microbial community within rodent models. Gut Microbes 3: 234-249.

Wos-Oxley, M.L., Plumeier, I., von Eiff, C., Taudien, S., Platzer, M., Vilchez-Vargas, R. et al. (2010) A poke into the diversity and associations within human anterior nare microbial communities. ISME J 4: 839-851.

Yan, M., Pamp, Sünje J., Fukuyama, J., Hwang, Peter H., Cho, D.-Y., Holmes, S., and Relman, David A. (2013) Nasal microenvironments and interspecific interactions influence nasal microbiota complexity and S. aureus carriage. Cell Host Microbe 14: 631-640. 
Table 1. Summary of patient history

\begin{tabular}{|c|c|c|c|c|}
\hline \multirow{2}{*}{ Factors } & \multirow{2}{*}{$\begin{array}{l}\text { CRS- } \\
\text { free }\end{array}$} & \multirow[t]{2}{*}{ CRS } & \multicolumn{2}{|c|}{ CRS phenotype } \\
\hline & & & CRSsNP & CRSwNP \\
\hline number of individuals recruited & 37 & 42 & 27 & 15 \\
\hline age $(\text { median }+ \text { range })^{a}$ & $\begin{array}{c}31 \\
(18-65)\end{array}$ & $\begin{array}{c}52 \\
(20-79)\end{array}$ & $\begin{array}{c}47 \\
(20-79)\end{array}$ & $\begin{array}{c}52 \\
(20-77)\end{array}$ \\
\hline $\begin{array}{c}\text { gender }^{\mathrm{b}} \text { - female } \\
\text { - male }\end{array}$ & $\begin{array}{l}18 \\
19\end{array}$ & $\begin{array}{l}11 \\
31\end{array}$ & $\begin{array}{c}9 \\
18\end{array}$ & $\begin{array}{c}2 \\
13\end{array}$ \\
\hline \multicolumn{5}{|l|}{ diagnosed medical condition of the nose ${ }^{c}$} \\
\hline $\begin{array}{r}\text { deviated nasal septum and inferior turbinate } \\
\text { hypertrophy } \\
\text { trauma to the nasal bone }\end{array}$ & 7 & 2 & 0 & 2 \\
\hline \multicolumn{5}{|l|}{ known allergies and asthma ${ }^{d}$} \\
\hline allergic rhinitis & 11 & 12 & 7 & 5 \\
\hline aspirin exacerbated respiratory disease & 0 & 3 & 0 & 3 \\
\hline hymenoptera venom allergy & 0 & 1 & 1 & 0 \\
\hline allergy to drugs & 6 & 3 & 2 & 1 \\
\hline allergy to foods & 1 & 2 & 2 & 0 \\
\hline asthma & 2 & 4 & 0 & 4 \\
\hline reported to be currently taking medications ${ }^{\mathrm{e}}$ & 13 & 23 & 14 & 9 \\
\hline 1 medication & 8 & 12 & 7 & 5 \\
\hline 2 medications & 2 & 6 & 3 & 3 \\
\hline 3 medications & 1 & 3 & 2 & 1 \\
\hline 5-6 medications & 2 & 2 & 2 & 0 \\
\hline \multicolumn{5}{|l|}{ medications $^{\text {f }}$} \\
\hline nasal steroids & 3 & 7 & 4 & 3 \\
\hline bronchial steroids & 1 & 3 & 1 & 2 \\
\hline asthma spray & 1 & 1 & 0 & 1 \\
\hline smoking (declared) ${ }^{\mathrm{g}}$ & 8 & 11 & 9 & 2 \\
\hline$<15$ per day & 2 & 5 & 5 & 0 \\
\hline$>15$ per day & 6 & 5 & 3 & 2 \\
\hline not specified & 0 & 1 & 1 & 0 \\
\hline MRSA positive & 0 & 2 & 1 & 1 \\
\hline
\end{tabular}

${ }^{\mathrm{a}}$ The Mann-Whitney test (two-tailed, unpaired) and the unpaired t-test assuming equally variance

(two-tailed) and also not assuming equal variance (with Welch's correction) were used to compare ages between the CRS group and the CRS-free group returning p-values of 0.0006 (Mann-Whitney $\mathrm{U}=434), 0.0005(\mathrm{t}=3.657, \mathrm{df}=77)$ and $0.0004(\mathrm{t}=3.711, \mathrm{df}=76.21)$, respectively, deemed to be highly significant different. The CRS-free group overall comprises more younger individuals, probably due to 
the fact that this control group were mostly having surgeries for innate deformations (like deviated nasal septum).

${ }^{\mathrm{b}}$ The Fisher's exact test (two-tailed, confidence intervals at 95\%) was used to compare the gender proportions between the CRS group and the CRS-free group returning a p-value of 0.0606, close to the alpha value set at $<0.05$, but deemed not to be significantly different.

${ }^{\mathrm{c}}$ no formal tests were performed here because it was clear that the reasons for nasal surgery differed between the CRS-free groups and the CRS groups.

${ }^{d}$ The Chi-square test (confidence intervals at 95\%) was used to compare proportions of individuals with different allergies between the CRS group and the CRS-free group returning a p-value of 0.3518 (chi-square $=5.557, \mathrm{df}=5$ ), deemed not to be significant different if considering an alpha value of $<0.05$.

${ }^{\text {e }}$ The Fisher's exact test (two-tailed, confidence intervals at 95\%) was used to compare the proportions of individuals taking medication between the CRS group and the CRS-free group returning a p-value of 0.1132 , deemed not to be significant different if considering an alpha value of $<0.05$. In addition, the Chi-square test (confidence intervals at 95\%) was used to compare proportions of individuals taking different numbers of medications between the CRS group and the CRS-free group returning a $\mathrm{p}$-value of 0.7752 (chi-square $=1.108, \mathrm{df}=3$ ), deemed not to be significant different if considering an alpha value of $<0.05$.

${ }^{\mathrm{f}}$ The Chi-square test (confidence intervals at 95\%) was used to compare proportions of individuals taking different types of medications between the CRS group and the CRS-free group returning a pvalue of 0.8158 (chi-square $=0.4073, \mathrm{df}=2$ ), deemed not to be significant different if considering an alpha value of $<0.05$.

${ }^{g}$ The Fisher's exact test (two-tailed, confidence intervals at 95\%) was used to compare smoking habits between the CRS group and the CRS-free group returning a p-value of 0.7929 , deemed not to be significant different if considering an alpha value of $<0.05$. 


\section{Figure legends}

Fig. 1. Difference between global bacterial community structures of all 79 swabbed individuals as analyzed by non-metric multidimensional scaling (nMDS), where each nMDS plot represents an anatomical region of the nasal passage: (A) anterior vestibule; (B) posterior vestibule; (C) inferior meatus and (D) middle meatus. The SIMPROF test associated with group-average agglomerative hierarchical clustering (using significance level of 1\%; 1000 permutations for mean profile; 999 simulation permutations; and the Bray-Curtis algorithm for comparison between samples) was used to delineate groups of individuals exhibiting statistically similar global bacterial community profiles. Within each plot, the colored dots (red, blue, green, purple, orange and aqua blue) represent the predominant groups of individuals $(\mathrm{n}=>7)$, where the gray dots represent those individuals that did not form predominant clusters. The letter above each dot denotes its delineated group.

Fig. 2. Mean relative abundance of those phylotypes discerned to constitute similarity among individuals within each of the predominant groups (as revealed in Fig. 1), where the SIMPER algorithm was used following the delineation of statistically determined groups of individuals from the SIMPROF analysis associated with group-average agglomerative hierarchical clustering.

Fig. 3. Differences in global bacterial community structures of swab and tissue biopsies obtained from 73 individuals as assessed by non-metric multidimensional scaling (nMDS). Each nMDS plot represents an anatomical region of the nasal passage: (A) inferior meatus and (B) middle meatus, where swabs are represented by black dots and tissue biopsies 
represented by gray dots. The global bacterial assemblages between the groups are statistically separable and significantly different (using ANOSIM and PERMANOVA).

Fig. 4. Taxonomic distinctness and evenness of communities derived from swabs and tissue biopsy samples. Funnel plots chart the average taxonomic distinctness (delta + ) and variation in taxonomic distinctness (lambda + ) against the number of phylotypes within each sample of the inferior meatuses (A-B) and middle meatuses (C-D), where swabs are represented by black dots and tissue biopsies represented by gray dots. Delta + and lambda + between swab and tissue samples were statistically significantly different (Welsh's t-test, p-value $=<0.05$ ).

Fig. 5. Global bacterial community structures of CRS and CRS-free individuals. Differences were visualized using non-metric multidimensional scaling (nMDS): (A) microbial community structures of CRS-free individuals at the 4 swabbed sites (white diamonds) versus those sites from individuals suffering from CRS (black diamonds); (B) microbial community structures of individuals suffering from CRSsNP at the 4 swabbed sites (white squares) versus those sites from individuals suffering from CRSwNP (black squares). No significant differences between global community assemblages were observed between any a priori groups (using PERMANOVA).

Fig. 6. Taxonomic distinctness and evenness of communities at the 4 swabbed sites derived from CRS and CRS-free individuals. Funnel plots chart the average taxonomic distinctness $($ delta +$)$ and variation in taxonomic distinctness (lambda + ) against the number of phylotypes within each swabbed sample: (A-B) CRS-free individuals (white diamonds) versus CRS individuals (black diamonds); and (C-D) CRSsNP individuals (white squares) versus CRSwNP individuals (black squares). Delta + and lambda + between these groups (CRS vs 
CRS-free) and (CRSsNP vs CRSwNP) were not statistically significantly different (Welsh's ttest, $\mathrm{p}$-value $=>0.05)$. 


\section{Supplementary Information}

\section{Table S1 (as pdf)}

Those phylotypes that have the greatest contribution towards the observed difference between the tissue biopsies and swabs of both anatomical regions (the middle meatuses and the inferior meatuses) in individuals with and without CRS. The discernible PTs were derived using the Similarity Percentage (SIMPER) algorithm in the PRIMER program, setting a cut-off at $70 \%$ cumulative contribution, in order to give only the top few PTs that contribute to the difference between the groups.

Fig. S1 (as tif)

Regions of the nasal passage

Fig. S2 (as tif)

Rarefaction curves portraying the number of resolved phylotypes against sequencing depth of each sample of the (A) anterior vestibule (swabbed), (B) posterior vestibule (swabbed), (C) inferior meatus (swabbed), (D) inferior meatus (tissue biopsies), (E) middle meatus (swabbed) and (F) middle meatus (tissue biopsies).

Fig. S3 (as tif)

The relative percent abundance of the 20 most abundant and prevalent phylotypes across all 462 samples.

Fig. S4 (as tif) 
Differences in the global bacterial community structures of 79 individuals (including the four anatomical regions along the nasal passage), as assessed by non-metric multidimensional scaling (nMDS), where (A) all 316 swabbed samples are ordinated on the plot, (B) only CRSfree individuals are ordinated, and (C) only CRS individuals are ordinated.

Fig. S5 (as tif)

Measures of species diversity, richness and taxonomic distinctness of samples, where mean value and standard deviation are plotted for each of the groups of interest (CRS-free, CRSsNP and CRSwNP) at each nasal region/sampling strategy. No statistical significant differences exist among the comparisons made between CRS and CRS-free individuals, or between CRSsNP and CRSwNP groups (p-value $>0.05$ ).

Fig. S6 (as tif)

$k$-dominance plots presenting cumulative ranked abundances plotted against species rank depicting bacterial phylotype diversity and dominance, comparing swab (blue) and tissue biopsies (red) from both the (A) inferior meatus and (B) middle meatus.

Dataset S1 (as xlsx)

Relative phylotype abundance across all 462 samples.

\section{Dataset S2 (as xlsx)}

Nucleotide sequences of all 447 phylotypes determined using Illumina-based amplicon deepsequencing and their phylogenetic assignment. 